\section{Apolipoprotein J, Sialinsäure-defizientes}

T. Arndt

Bioscientia Institut für Medizinische Diagnostik $\mathrm{GmbH}$, Ingelheim, Deutschland

Synonym(e) Sialinsäure-defizientes Apolipoprotein J

Englischer Begriff apolipoprotein J, sialic acid-deficient; apolipoprotein J, sialic-acid index; SIJ

Definition Jene Isoformen des Apolipoproteins J (Apo-J), die unter chronischem Alkoholmissbrauch in erhöhter Konzentration im Plasma vorliegen.

Beschreibung Apolipoprotein J ist Bestandteil der $>$ High Density Lipoproteine (HDL). Es ist physiologisch etwa 7-mal stärker sialyliert als $\triangleright$ Transferrin. Eine durch Alkoholkonsum induzierte Sialylierungsstörung des Apo-J könnte deshalb chronischen Alkoholmissbrauch ( $\triangleright$ Alkoholmissbrauchskenngrößen) empfindlicher als das $\triangleright$ carbohydrate-deficient transferrin (CDT) anzeigen. Tatsächlich war der Sialylierungsgrad von Apolipoprotein J nach Aufnahme von $60 \mathrm{~g}$ - Ethanol/Tag an 30 aufeinanderfolgenden Tagen um $50 \%$ erniedrigt. Es wurde eine hohe diagnostische Sensitivität ( $\vee$ Sensitivität, diagnostische) und Spezifität ( $\vee$ Spezifität, diagnostische) zur Kontrolle von Abstinenz und Rückfällen während Alkoholentzugstherapie beschrieben. Weiterführende klinische Studien blieben aus. Der Parameter wurde nicht zur Routinetauglichkeit geführt.

\section{Literatur}

Ghosh P, Hale EA, Lakshman MR (2001) Plasma sialic-acid index of apolipoprotein J (SIJ): a new alcohol intake marker. Alcohol 25:173-179 